\title{
Functional IBV Minigenomes Generated by Recombinant Fowl Pox Viruses for use in IBV- Targeted Recombination Studies
}

\author{
SHARON EVANS, DAVID CAVANAGH, AND PAUL BRITTON \\ Institute for Animal Health, Compton, Newbury, Berkshire, England
}

\section{INTRODUCTION}

Having previously demonstrated that the IBV defective (D)-RNA CD-61, can be used as an RNA vector for the expression of heterologous genes via electroporation of transcripts into infected cells (Stirrups et.al., 2000b) we wished to improve this procedure with another strategy. Hence, IBV D-RNA sequences (minigenomes) were introduced into the fowl pox virus (FPV) genome for use in a FPV/T7 system. Modified CD-61 D-RNA minigenomes were generated in situ, in a helper virus dependent manner, from cells coinfected with IBV, recombinant FPV containing CD-61 directed by the T7 promoter and rFPV expressing T7 RNA polymerase. Efficient rescue of CD61 D-RNAs by helper IBV was demonstrated upon serial passage in tissue culture.

For recombination purposes, minigenomes (D-RNAs) require stable expression of extraneous sequences. Therefore, CD-61 was modified to contain the reporter gene luciferase (LUC). Recombinant FPV containing CD-61LUC was assessed in $\mathrm{rFPV} / \mathrm{T} 7$ rescue studies for production of the DRNA and expression of luciferase. In addition, we demonstrated the rescue of rFPV-generated CD-61 using the heterologous IBV strain M41. 


\section{RESULTS AND DISCUSSION}

We have already demonstrated that in vitro T7-derived D-RNA transcripts of CD-61 can be replicated and packaged (rescued) in a helper virusdependent manner (Penzes et al., 1994). Hence, CD-61 cDNA was modified by the addition of the hepatitis delta virus ribozyme plus $\mathrm{T} 7$ terminator, downstream of the 3' UTR, prior to cloning within the FPV transfer vector pEFL10 (Fig. 1). FPV-specific flanking sequences allow for homologous recombination of the CD-61 cassette into the genome of FPV. The LUC gene, under the control of IBV gene 5 transcription associated sequence (TAS; Hiscox et.al., 1995) was cloned into the Pma CI site in domain III of CD-61.

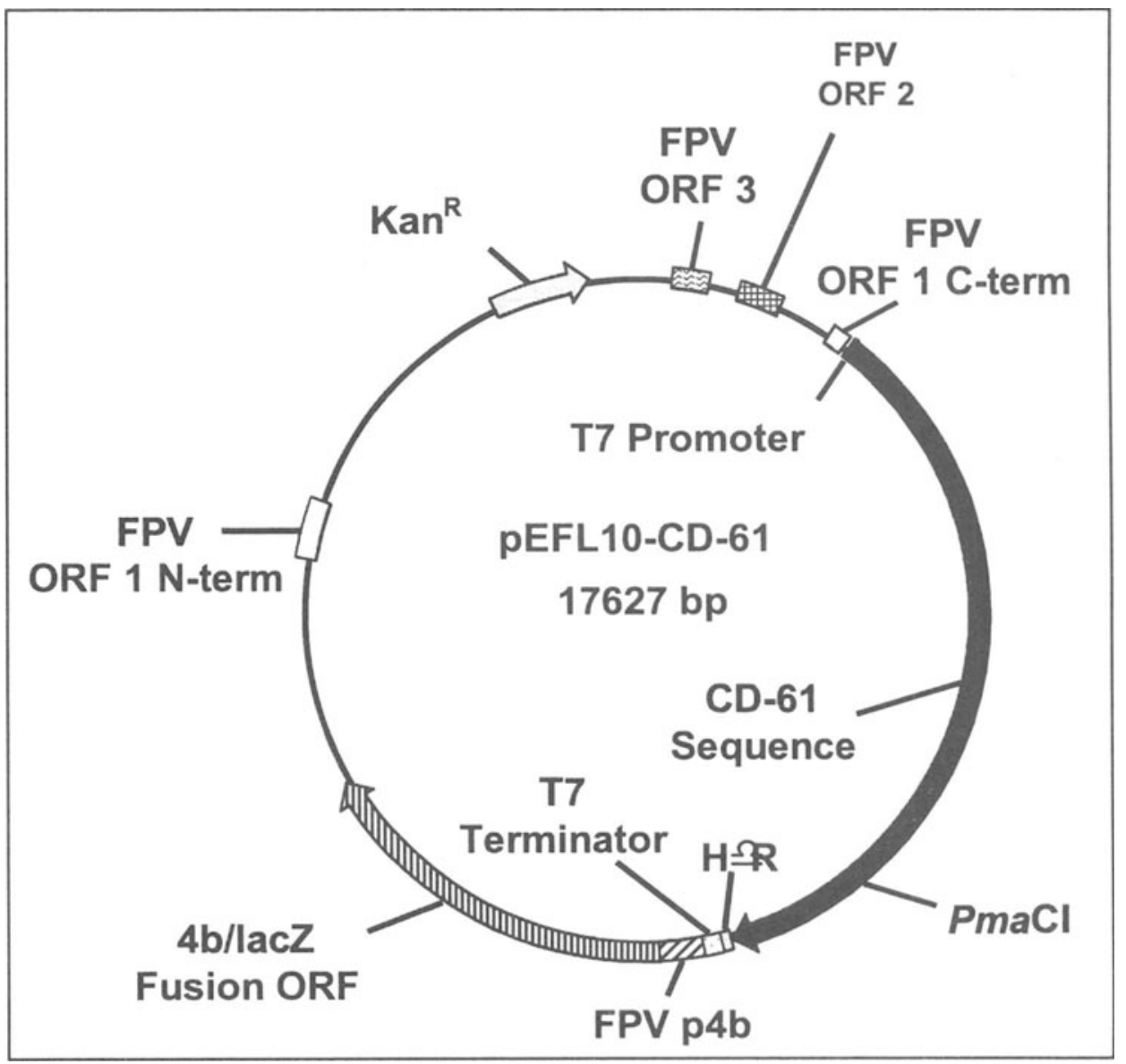

Figure 1. Schematic showing salient features of FPV transfer vector containing modified CD61 D-RNA. 
For rescue studies, chick kidney $(\mathrm{CK})$ cells were co-infected with IBV (Beaudette), rFPV expressing T7 RNA polymerase and $\mathrm{rFPV/CD}-61$. Serial passage of virus supernatants was follwed by total RNA extractions. Northern blot analysis of IBV-specific RNAs showed CD-61 D-RNA, which was rescued from passage 1 (P1) onwards. Similar experiments using Vero cells also demonstrated rescue of CD-61 D-RNA. Mammalian Vero cells do not support productive FPV infection, which demonstrates that the helper IBV has replicated and packaged the D-RNA; the presence of CD-61 in vero cell lysates is not the result of rFPV transmission.

Following efficient rescue of the IBV D-RNA in the $\mathrm{rFPV} / \mathrm{T} 7$ system, we wished to assess the potential of $\mathrm{FPV} / \mathrm{CD}-61$ for expression of heterologous genes. Recombinant FPV/CD-61LUC (containing the luciferase reporter gene) was isolated and analysed in rescue experiments. Extracted RNAs from $\mathrm{CK}$ cells co-infected with IBV, $\mathrm{rFPV} / \mathrm{T} 7$ and $\mathrm{rFPV} / \mathrm{CD}-61 \mathrm{LUC}$ were Northern blotted and hybridised to ${ }^{32} \mathrm{P}$-labelled LUC-specific DNA. CD61LUC D-RNA was detected from P1 and a potential LUC-specific mRNA was also present.

Infected cell lysates from $\mathrm{rFPV/CD-61LUC} \mathrm{rescue} \mathrm{passages} \mathrm{were} \mathrm{assayed}$ for expression of luciferase. Luciferase activity was observed from $\mathrm{P} 0$, showing a marked increase to $\mathrm{P} 3 / \mathrm{P} 4$. Therafter, the activity declined to background levels by $\mathrm{P} 7 / \mathrm{P} 8$. In previous studies, in vitro $\mathrm{T} 7$ transcripts of CD-61LUC were introduced into cells by electroporation prior to rescue. In this case luciferase levels were 200-fold less than for rFPV/CD-61LUC rescue. This demonstrates that the rFPV system supports expression of a reporter gene from the IBV D-RNA and is significantly more efficient than electroporation techniques.

The LUC-specific D-RNA-derived mRNA was amplified by RT.PCR prior to direct sequencing of the 5' end. Results identified the IBV leader sequence fused to the TAS site of gene 5, proximal to the LUC gene. Moreover, confirming the presence of an IBV-transcribed LUC-specific mRNA, as shown by Northern blotting.

Previous work has demonstrated rescue of CD-61, following electroporation of T7 transcripts, by heterologous IBV strains (Stirrups et.al., 2000a). Consequently, we used IBV strain M41 as helper virus in the rFPV system. Total RNA from co-infection of CK cells with M41, rFPV/T7 and rFPV/CD-61 was assessed by Northern blotting. Results indicated that CD61 D-RNA, derived from rFPV/CD-61, was rescued by IBV M41 from P2.

RT.PCR analysis of the D-RNA was used to sequence the 5' ends of the D-RNA rescued by heterologous helper IBV. The M41-rescued CD-61 had acquired the genomic leader sequence corresponding to the 5' of the M41 helper virus. Sequences downstream of the leader junction corresponded to the original CD-61 Beaudette sequence, confirming that leader switching 
(Makino \& Lai, 1989) occurred during rescue of CD-61 by M41. Furthermore, the leader switching event proves that rFPV/CD-61-derived DRNA is transcribed and replicated by M41 IBV.

We have shown that fowl pox virus recombinants can improve delivery of D-RNA minigenomes to IBV-infected cells, which are replicated and packaged by the helper virus. This is expected to improve the opportunity for recombination between D-RNA containing a target gene and the helper IBV genome.

\section{ACKNOWLEDGEMENTS}

This work was supported by the Ministry of Agriculture, Fisheries and Food, UK, Project code OD1905 and by grant number CT950064 of the Fourth RTD Framework Programme of the European Commission (EC). S. Evans was supported by a BBSRC Realising Our Potential Award (ROPA).

\section{REFERENCES}

Hiscox, J. A.., Mawditt, K. L., Cavanagh, D. \& Britton, P. (1995). Investigation of the control of coronavirus subgenomic mRNA transcription by using T7-generated negative sense RNA transcripts. J. Virol .69, 6219-6227.

Makino, S. \& Lai, M. M. C. (1989). High-frequency leader sequence switching during coronavirus defective interfering RNA replication. J. Virol .63, 5285-5292.

Penzes, Z., Tibbles, K., Shaw, K., Britton, P., Brown, T. D. K. \& Cavanagh, D. (1994). Characterisation of a replicating and passaged defective RNA of avian coronavirus infectious bronchitis virus. Virology. 203, 286-293.

Stirrups, K., Shaw, K., Evans, S., Dalton, K., Cavanagh, D. \& Britton, P. (2000a). Leader switching occurs during the rescue of defective RNAs by heterologous strains of the coronavirus infectious bronchitis virus (IBV). J. Gen. Virol .81, 791-801.

Stirrups, K., Shaw, K., Evans, S., Dalton, K., Casais, R., Cavanagh, D. \& Britton, P. (2000b). Expression of reporter genes from the defective RNA CD-61 of the coronavirus infectious bronchitis virus. J. Gen. Virol. 81, 1687-1698. 